\title{
The relationship between oral, the kidney and urinary tracts infections and preterm delivery in pregnant women admitted to educational hospitals, Isfahan, Iran
}

\author{
Mahboubeh Valiani ${ }^{1}$, Fatemeh Torabi ${ }^{1 *}$, Fatanat Sadat khodaei ${ }^{1}$ \\ 1. School of Nursing and Midwifery, Isfahan University of Medical Sciences, Isfahan, Iran
}

Received: 5 August 2021

Accepted for publication: 2 October 2021

[EPub a head of print-18 October 2021]

Payesh: 2021; 20 (5): 581- 587

\begin{abstract}
Objective (s): The most common cause of death and mortality of infants is preterm labor. The identification of factors associated with preterm labor will reduce preterm infant mortality. This study aimed to determine the relationship between preterm delivery and oral, kidney and urinary tracts infections in pregnant women

Methods: This was a case-control study that carried on the 198 pregnant women (66 pregnant women with premature labor as case group and 132 pregnant women with term delivery as control group) in selected hospitals in Isfahan, Iran, in 2018. For data collection we used demographic and fertility questionnaire, and the Holbrook standard checklist. Data were analyzed using descriptive statistics and t-test, chi-square, Mann-Whitney, Fisher's exact test, logistic regression.

Results: The results showed that there was no significant relationship between preterm labor and kidney, urinary tracts infections $(p=0.626)$. While the logistic regression showed significant association between the onset of uterine contractions (preterm birth) and of the mouth and teeth infections $(\mathrm{p}=0.012)$

Conclusion: The findings suggest that there is relationship between preterm deliveries and oral infections. Therefore, it is recommended that preventive necessary measures be taken or treat oral infections faster in women before and during pregnancy.
\end{abstract}

Keywords: Preterm Labor, Oral Infection, Holbrooke

\footnotetext{
* Corresponding author: School of Nursing and Midwifery, Isfahan University of Medical Sciences, Isfahan, Iran

E-mail: fattane_khodaei@yahoo.com
} 
نشريه يزوهشكده علوم بهداشتى جهاددانشكاهى

\title{
بررسى ارتباط عفونتهاى دهان و دندان و عفونتهاى كليه و مجارى ادرار با زايمان زودرس در مادران باردار بسترى در زايشكاه بيمارستانهاى آموزشى منتخب شهر اصفهان
}

\author{
محبوبه واليانى' ، فاطمه ترابى ؛ فتانه سادات خدايى ا* \\ I. دانشكده يرستارى مامايى، دانشكاه علوم يزشكى اصفهان، اصفهان، ايران

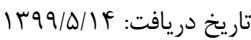

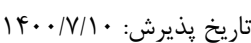

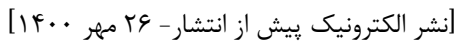

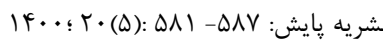

جكيده

مقدمه: زايمان زودرس به عنوان شايعترين علت مرى و مير نوزادان محسوب مىشود كه شناسايى عوامل مرتبط با آن مىتواند در جهت كاهش زايمانهاى

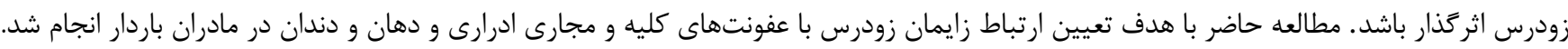

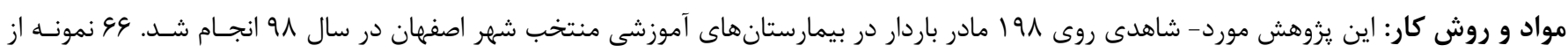

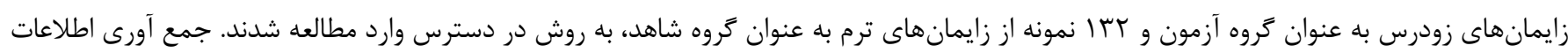

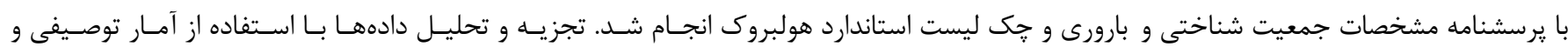

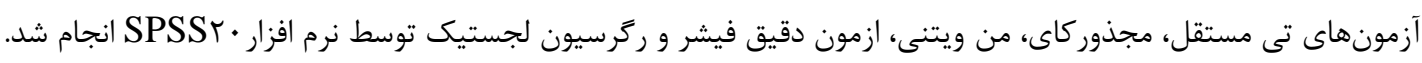

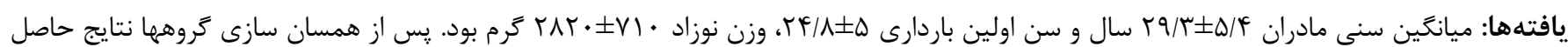

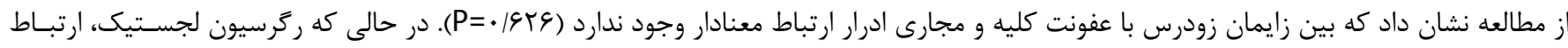

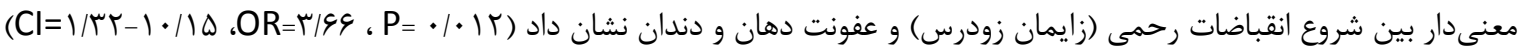

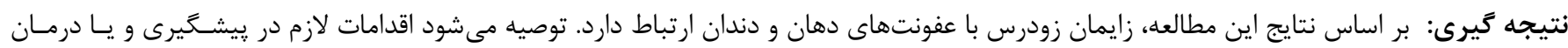

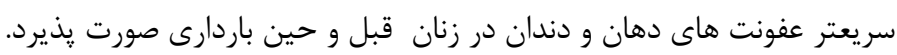
كليد وازهها: زايمان زودرس، عفونت دهان و دندان، هولبروى 
عفونت والتهاب است [IV] نظر به اين كه عوامل مرتبط بــا زايمـان

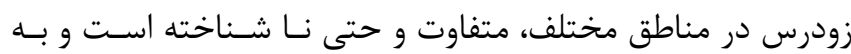

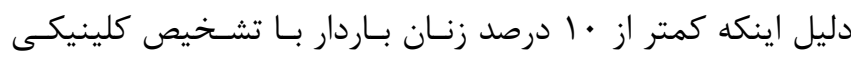

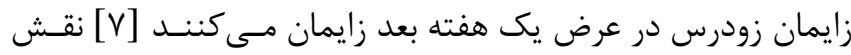

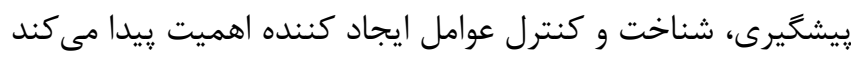
هم حنين با توجه به شيوع بالاى زايمان زودرس شايد با بِيشكيرى

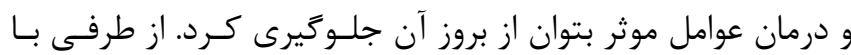

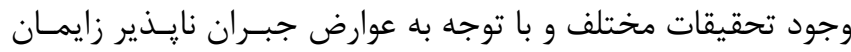

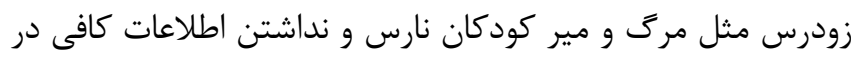
مورد عوامل و علل مرتبط بـا زايمـان زودرس در شـهر اصفههان (بـانـان

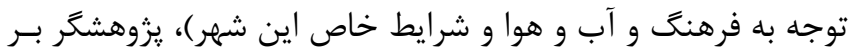

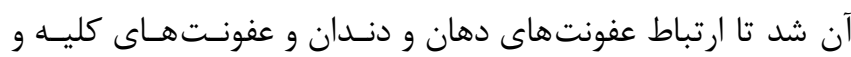
مجارى ادرار با زايمان زودرس مورد مطالعه قرار دهد.

\section{مواد و روش كار}

اين مطالعه برروى 191 مادر باردار انجـام شــد وسـطح معنسى دارى دوى

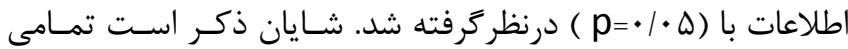

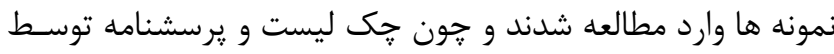

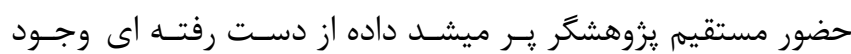

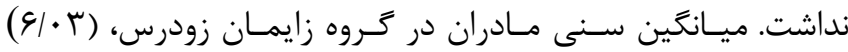

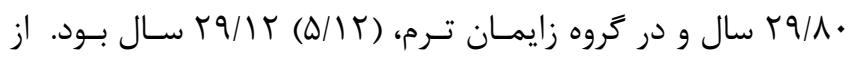
نظر سطح تحصيلات، در كروه زايمان ترم بيشترين فراوانى مشـاهده

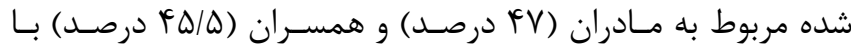

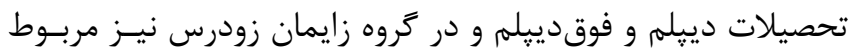

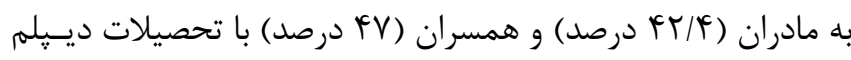
و فوقدييلم بوده است. 9/9 • درصد از مادران در كروه زايمان ترم و

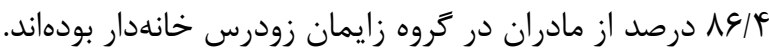

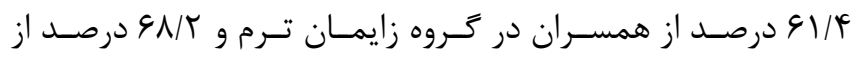

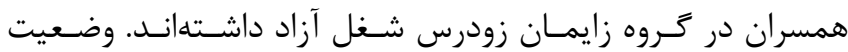

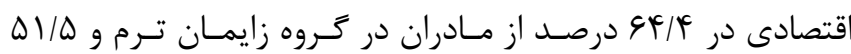

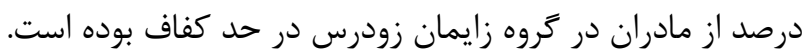

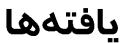

اين مطالعه برروى 191 مادر باردار انجـام شـد وسـطح معنسى دارى

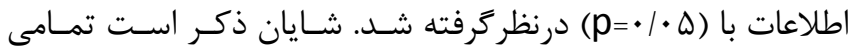

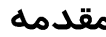

زايمان زودرس به ختم باردارى بيش از كامل شدن MV هفته يا قبل

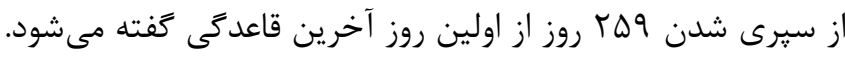

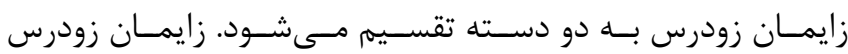

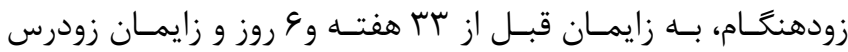

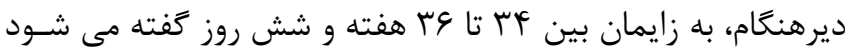

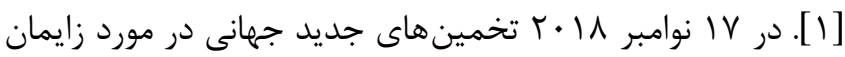

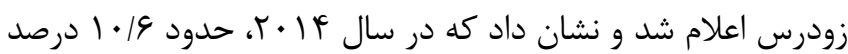
از كل زاد و ولد در سراسر جهان زودرس بودهاند [r]. نتايج مطالعهـ

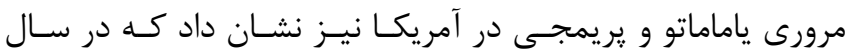

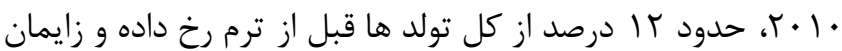

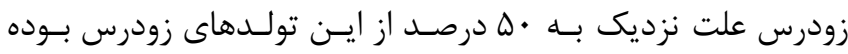

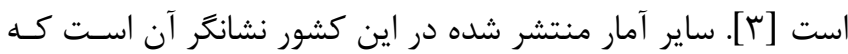

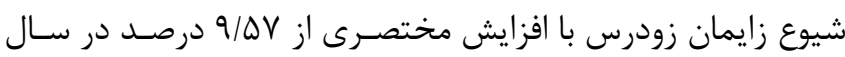

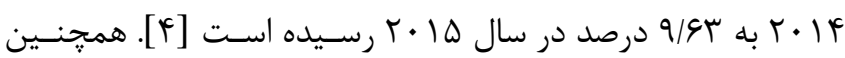

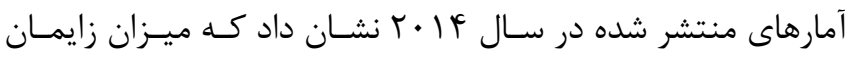

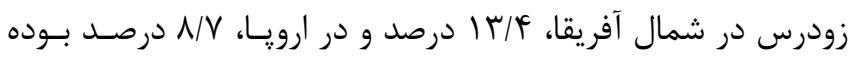

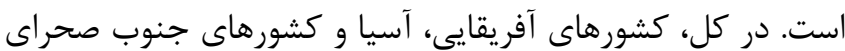

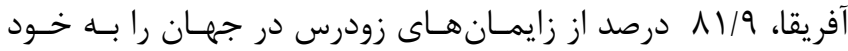

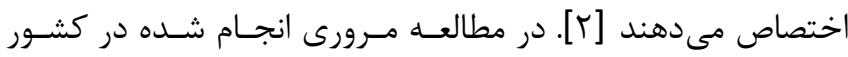
ايران، اين ميزان ب/9 درصد كزارش شده است [ه]. آمار بروز زايمان

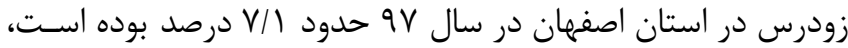

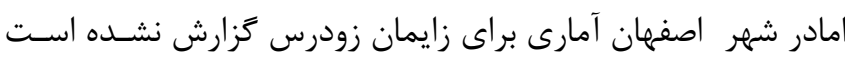

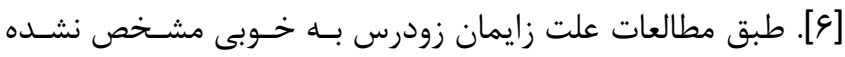

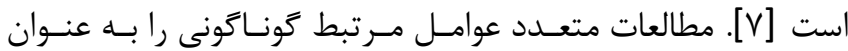

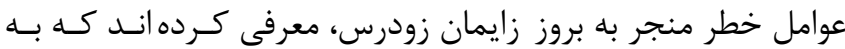
عنوان مثال مى توان به مواردى همانـــد وزن زيـاد و و جـاقى مـادران

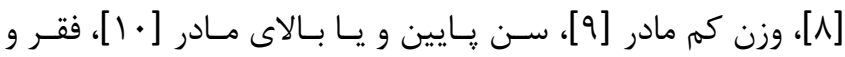
كمبود ويتامين سى [11]]، رفتارهاى ناسـالم و اسـترس و اضـطراب

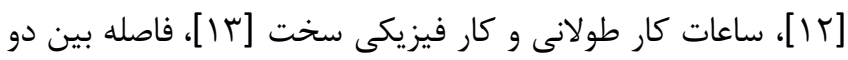

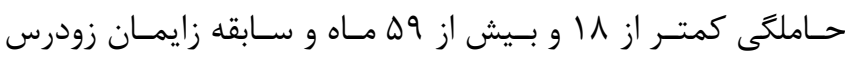

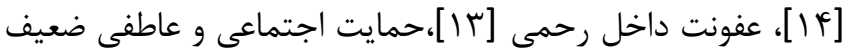

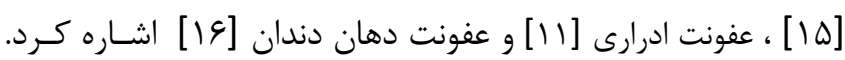

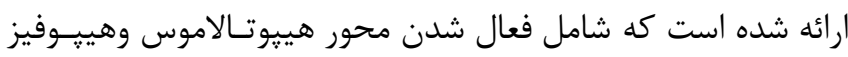

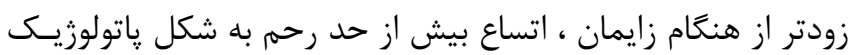

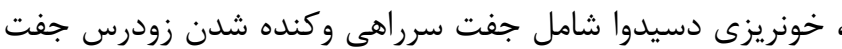


نشريه يزوهشكده علوم بهداشتى جهاددانشكاهى

درصد از مادران در ₹روه زايمان زودرس خانهدار بودهاند.

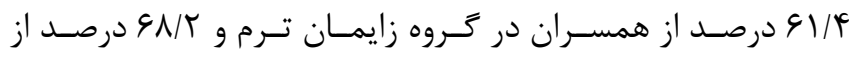

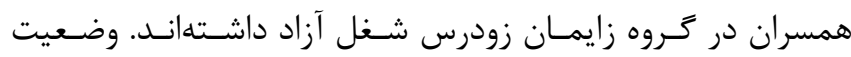

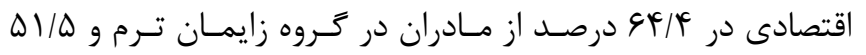
درصد از مادران در زروه زايمان زودرس در حد كفـاف بـوده اسـت.

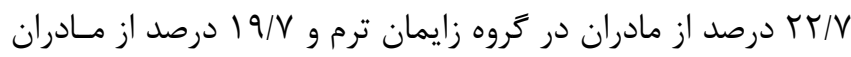
در گروه زايمان زودرس عفونت كليه و مجارى ادرارى داشتند.

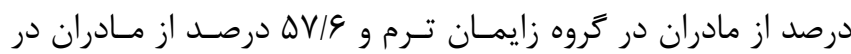

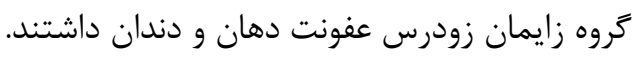

نمونه ها وارد مطالعه شدند و جون جك ليست و يرسشنامه توسـط

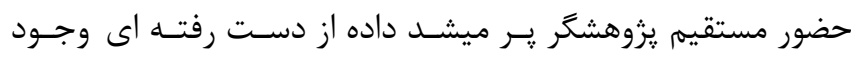

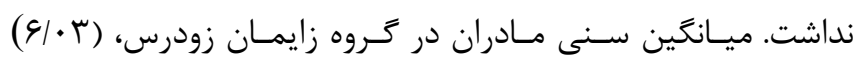
• سطح تحصيلات، در گروه زايمان ترم بيشترين فراوانى مشاهده شده

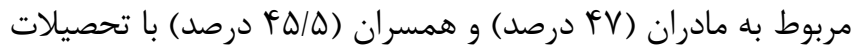
دييلم و فوقدييلم و در گروه زايمان زودرس نيز مربـوط بـهـ مـادران -

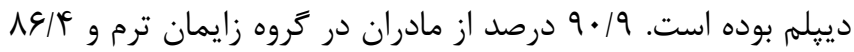

جدول ا: مقايسه توزيع فراوانى مشخصات بارورى واحدهاى يثوهش در دو كروه مورد و شاهد

\begin{tabular}{|c|c|c|c|c|c|}
\hline 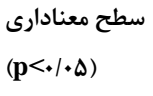 & آماره & 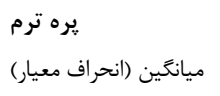 & ميانگين (انحراف معيار) & & \\
\hline.$/ 191$ & $\mathrm{t}=\cdot / 1 \mathrm{r \Lambda}$ & r|/q) $\pm(\Delta / r q)$ & $r r / \cdots \pm(r / r q)$ & & $\begin{array}{r}\text { سن ازدواج) } \\
\text { (سال }\end{array}$ \\
\hline . NRT & $\mathrm{t}=\cdot / \mu F f$ & $r \Delta / \cdots \pm(\Delta / \Lambda r)$ & $r F / V T \pm(Y / G q)$ & & سن اولين حاملكى (سال) \\
\hline$<\cdot / \cdot \cdot 1$ & $\mathrm{t}=1 \wedge .90$ & 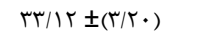 & $\Gamma \wedge / \wedge v_{ \pm}(1 / \cdot \wedge)$ & & سن باردارى (هفته) \\
\hline$\cdot 19 \cdot 9$ & $\mathrm{t}=-\cdot|\Delta| T$ & $\Delta / \Delta T \pm(\Psi / \cdot \varphi)$ & $\Delta / I T \pm(r / V \Delta)$ & & 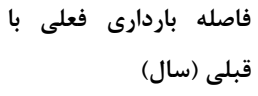 \\
\hline$<\cdot / \cdot \cdot 1$ & $\mathrm{t}=11 / 4 \varepsilon \Lambda$ & KITV/VT $\pm(\varepsilon \wedge \Lambda / r q)$ & $\mid V \wedge / q F \pm(F \cdot 1 / 9 \Delta)$ & & وزن نوزاد \\
\hline.$/ 19$ & $\chi^{2}=1 / v 11$ & $\begin{array}{l}r q(r q / 4) \\
r \cdot(\varphi \cdot \mid q)\end{array}$ & $\begin{array}{l}q \Delta(\uparrow q / r) \\
q V(\Delta \cdot / \Lambda)\end{array}$ & 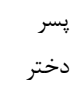 & جنس نوزاد \\
\hline & $\chi^{2}=r / \cdot s 4$ & $\begin{array}{l}r \Delta(\Delta T / \cdot) \\
r(\mathcal{L V} / \cdot)\end{array}$ & $\begin{array}{l}\Lambda F(G / G) \\
F \wedge(T G / F)\end{array}$ & سزبيعى & توع زايمان \\
\hline.$/ 9 T \mathrm{~V}$ & $\chi^{2}=\cdot 1 \cdot \cdot 1$ & $\begin{array}{l}r T(99 / V) \\
11(T / T)\end{array}$ & $r \Delta(T K / T)$ & سبيعى سزارين & $\begin{array}{r}\text { نوع زايمان قبلى } \\
\text { تعداد (درصد) }\end{array}$ \\
\hline$\cdot 1 \cdot \cdot 1$ & $\chi^{2}=r r / r v \Delta$ & $\begin{array}{l}10(Y K / V) \\
01(V V / T)\end{array}$ & $\begin{array}{l}r(T / r) \\
1 \% q(q V / V)\end{array}$ & ندارد - مارد & سابقه زايمان زودرس \\
\hline$\cdot / T \Delta \Delta$ & $\chi^{2}=1 / r q \Lambda$ & $\begin{array}{l}V(1 \cdot / 4) \\
\Delta 9(19 / 4)\end{array}$ & $\begin{array}{l}\Lambda(q / 1) \\
\operatorname{IrF}(9 / 9)\end{array}$ & ندارد & زودرس خانوادكى زايمان \\
\hline
\end{tabular}

جدول r: ارتباط زايمان زودرس با عفونت كليه و مجارى ادرار

\begin{tabular}{|c|c|c|c|c|c|}
\hline سطح معنادارى(ه+/>>p) & آماره & يره ترم & 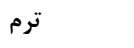 & & \\
\hline & & 19/V(1T) & $T r / V(r \cdot)$ & 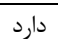 & عفونت كليه و مجارى ادرارى \\
\hline .1949 & $\chi^{2}=\cdot / r \mu \Lambda$ & $\Lambda \cdot / r(\Delta r)$ & $V V / r(1 \cdot r)$ & ندارد & \\
\hline
\end{tabular}

جدول سا: بر آورد ضريب ركرسيون لجستيك و نسبت شانس ارتباط عفونت دهان و دندان بر زايمان زودرس

\begin{tabular}{|c|c|c|c|c|}
\hline فاصله اطمينان ه 9٪ براى نسبت شانس & 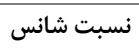 & سطح معنادارى & خطاى معيار & بر آورد ضر يب \\
\hline $1 / T r-1 \cdot / 10$ & r/\&\&f &.$/ \cdot 14$ & $\cdot / D T$ & $1 / 499$ \\
\hline
\end{tabular}


سال بيستم، شماره هنجم، مهر - آبان ••lf

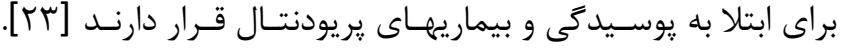

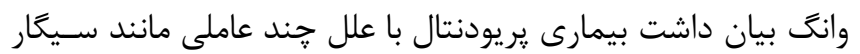

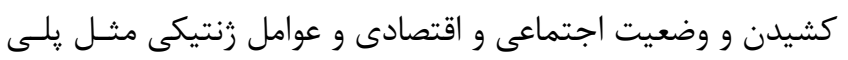
مورفيسم در زن هاى التهابى باعث آزاد شدن واسطه واى إسى التهابى در مادران مى شود و اين عوامل ممكن است باعث ايجاد زايمان زودرس

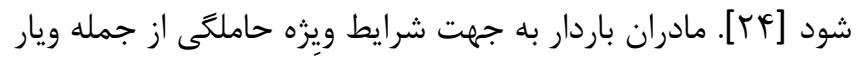

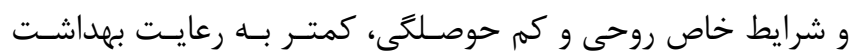

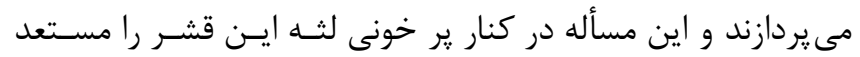

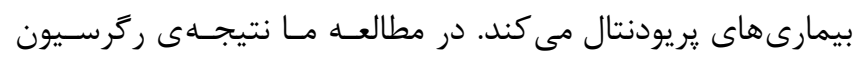

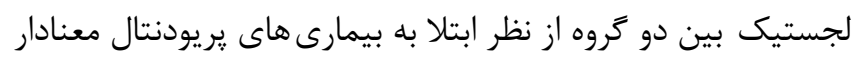

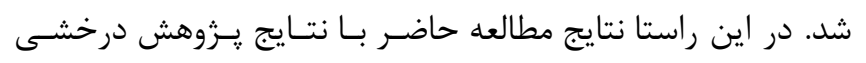

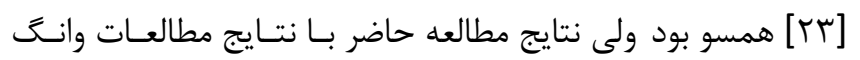

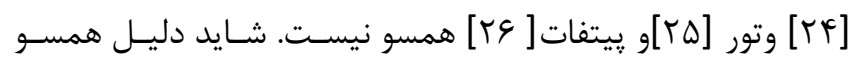

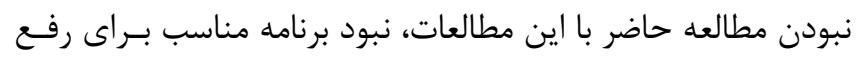

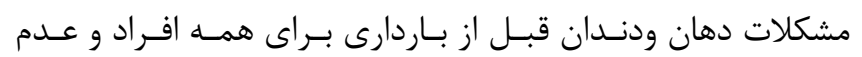

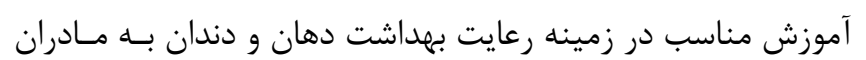

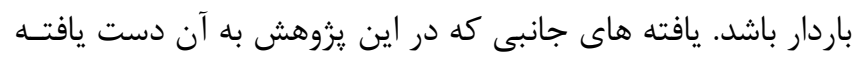

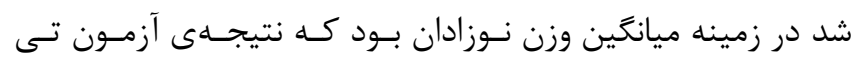

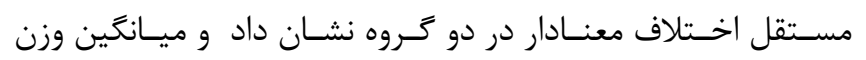

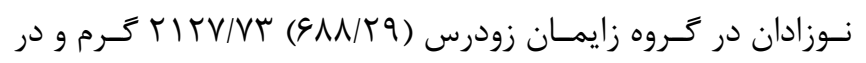

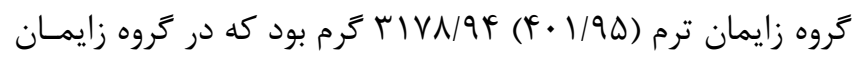

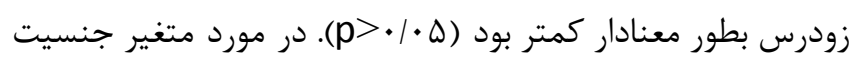

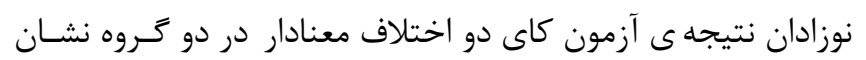

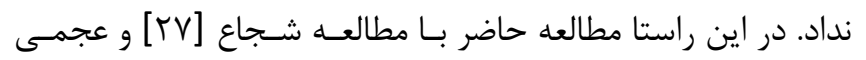

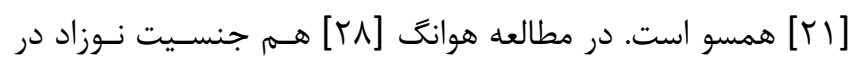

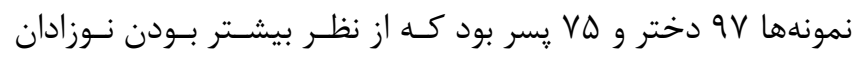

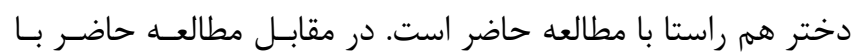

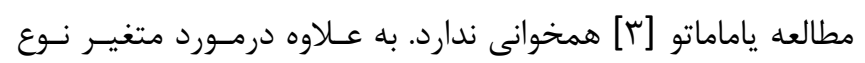

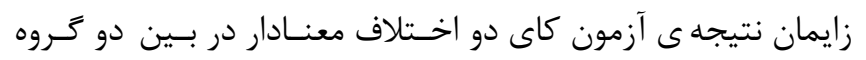

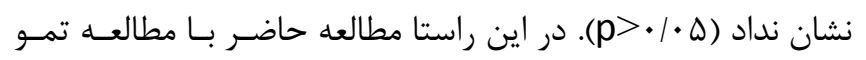

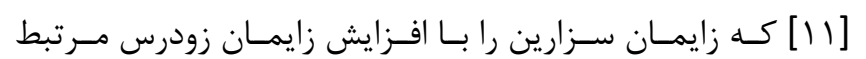

دانستند هم راستا نيست.

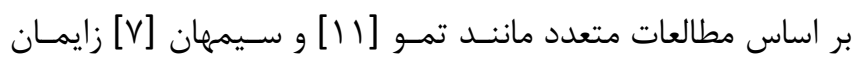

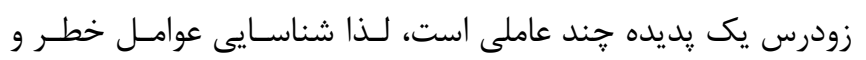

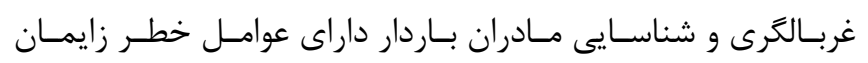

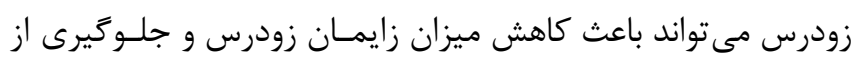

\section{بحث و نتيجه گيرى}

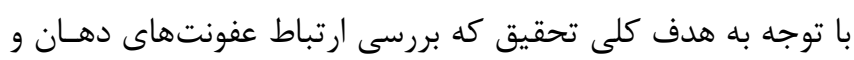

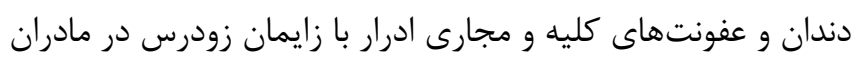

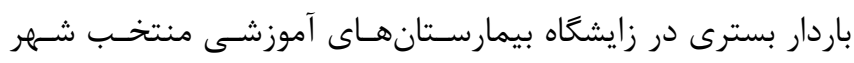

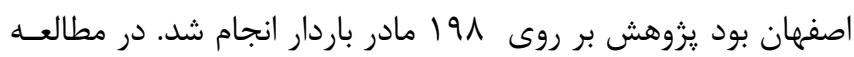

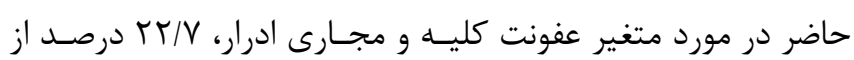

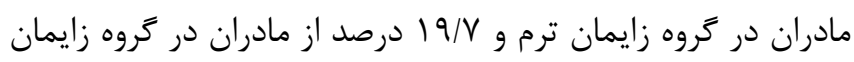

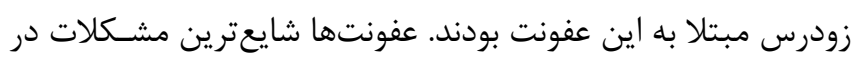

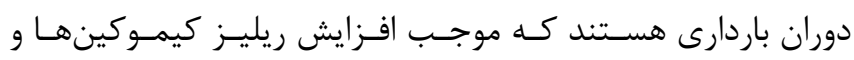

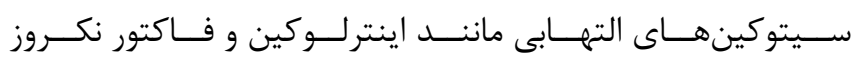

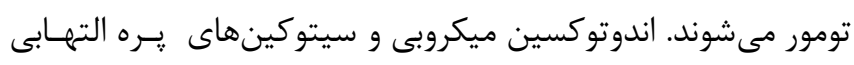

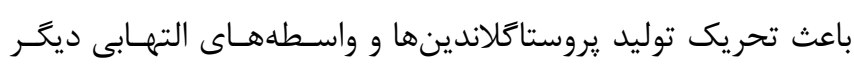

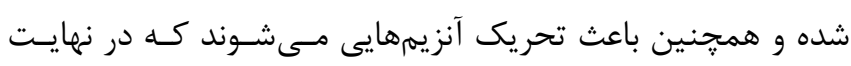

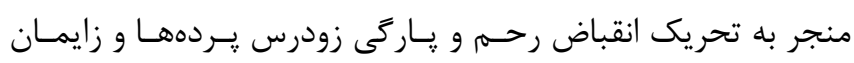

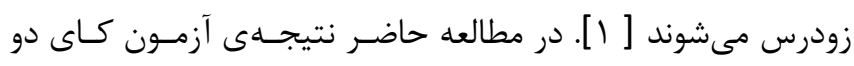

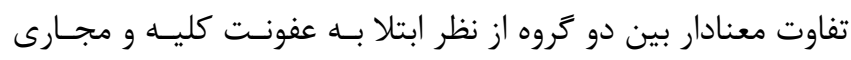

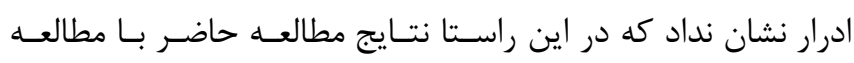

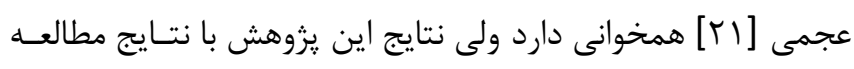

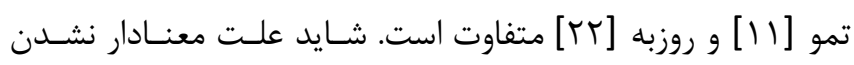

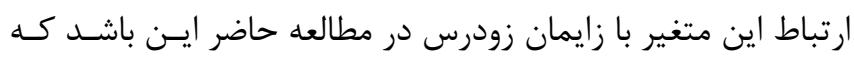

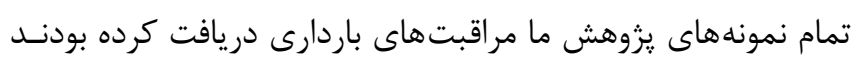

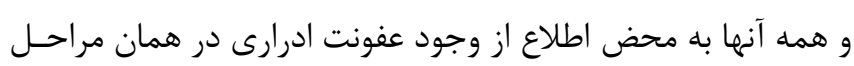

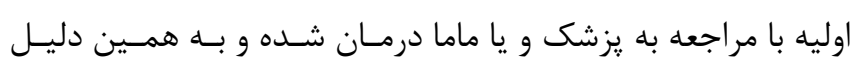

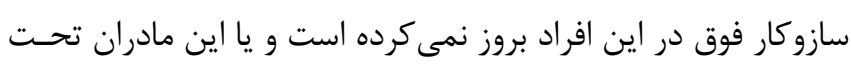

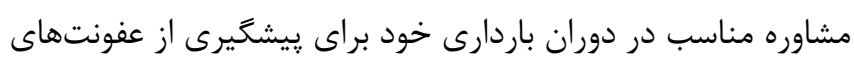

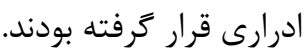

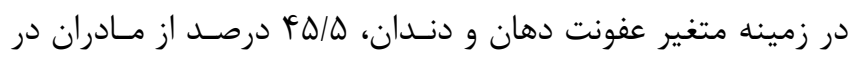

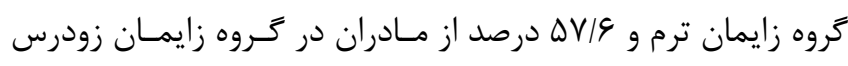

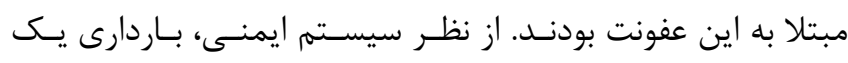

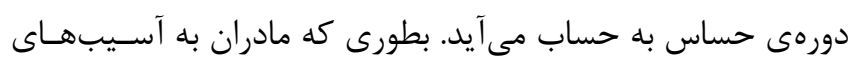

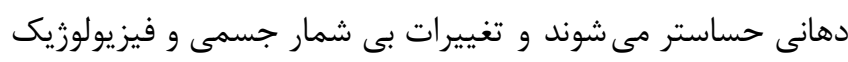

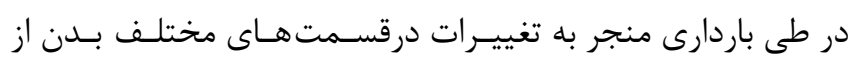

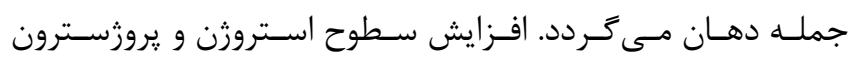

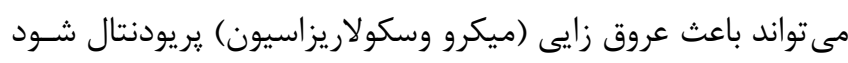

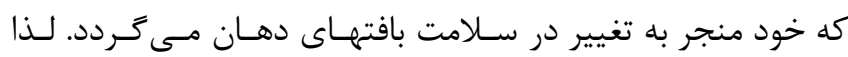

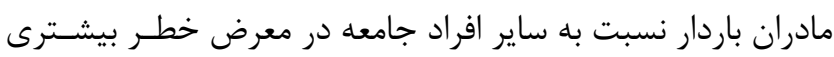


به آنها نمونه يزوهش نياز به بهره مندى از حافظه داشت، ممكن بود به صورت دقيق ياسخ داده نشود كه شناسايى آن ها خارج از كنترل

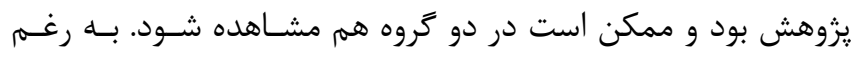

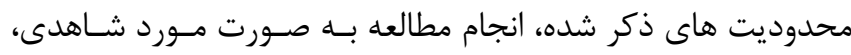

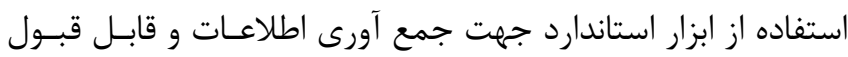
بودن حجم نمونه را ميتوان به عنوان نقاط قوت مطالعه مطرح نمود.

\section{سهم نويسند}

محبوبه واليانى: استاد راهنما، مجرى طرح، مشاركت در در تدوين مقان مقاله

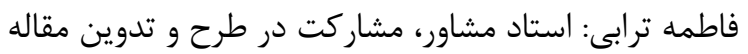

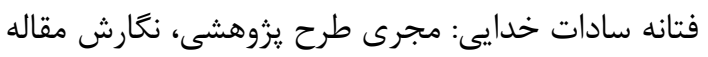

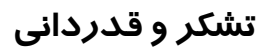

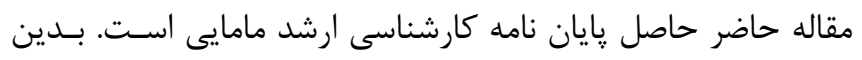

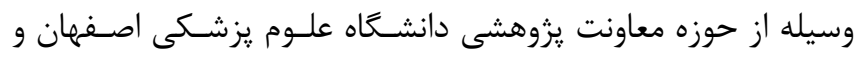

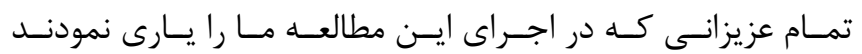
سياسگزارى مى گَردد.

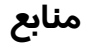

1. Cunningham G, Leveno KJ, Bloom SL, Spong CY, et al. Williams's obstetrics. 25th ed. new York: Appleton and lange, 2018;803-807

2. World Health Organization 2019، Available data in 2019/5/21

3. Yamamoto S, Premji S. The role of body, mind, and environment in preterm birth: mind the gap. Journal of Midwifery \& Women's Health 2017;62:696-705

4. Martin JA, Hamilton BE, Osterman MJK, Driscoll AK, Drake P. Births: Final Data for 2015. National Vital Statistics Reports 2017; 66: 1

5. Vakilian K, Ranjbaran M, Khorsandi M, Sharafkhani N, Khodadost M. Prevalence of preterm labor in Iran: A systematic review and meta-analysis. International Journal of Reproductive Bio Medicine 2017; 13: 743-748

6. http://sib.iums.ac.ir/home/login ‘Available data in 2019/5/21

7. Simhan HN. Management of Preterm Labor. American College of Obstetricians and Gynecologists. "Practice Bulletin No. 171". Obstetrics and Gynecology 2016; 128: 155-164

8. Cnattingius $\mathrm{S}$, Villamor $\mathrm{E}$, Johansson $\mathrm{S}$, Edstedt Bonamy AK, Persson M, Wikström AK, et al.

\begin{abstract}
تولد نوزادان نارس شود. علاوه بر اين در صورت وجود عوامل مستعد

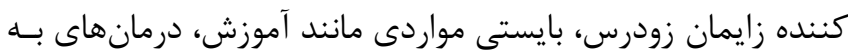

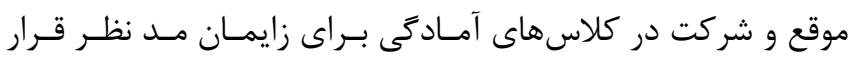

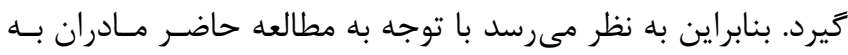

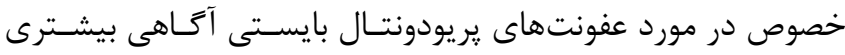

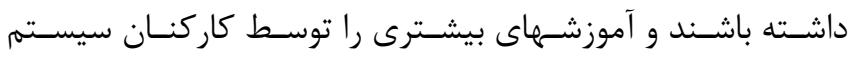
بهداشتى دريافت كنند. البته در اين مطالعه ما قادر به بررسى تمـام

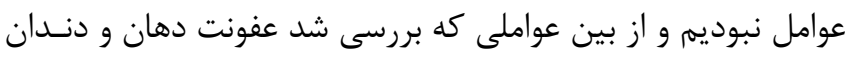

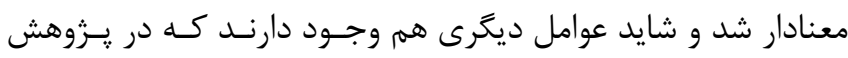

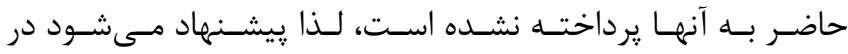

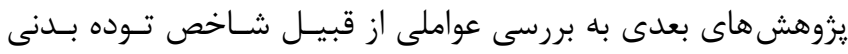

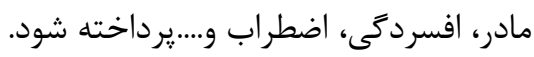

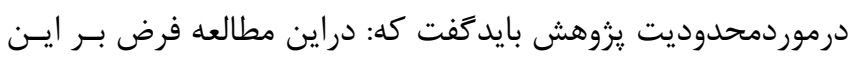
بود كه واحدهاى مورد يزوهش صادقانه به سوالات جواب مى دهى دهند،

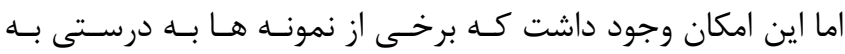
سوالات جواب ندهند. ازطرفى در مورد سوالاتى كه براى پاسخكويى إنى
\end{abstract}

Maternal Obesity and Risk of preterm Delivery.The Journal of the American Medical Association 2013; 22: $2362-2370$

9. Girsen AI, Mayo JA, Carmichael SL, Phibbs CS, Shachar BZ, Stevenson DK, et al. Women's prepregnancy underweight as a risk factor for preterm birth: a retrospective study. BJOG: An International Journal of Obstetrics \& Gynaecology 123.12. 2016; $112-21$

10. Laopaiboon $\mathrm{M}$, Lumbiganon $\mathrm{P}$, Intarut $\mathrm{N}$, Mori R, Ganchimeg T, Vogel JP, et al. Advanced Maternal Age and Pregnancy outcomes:a Multicountry Assessment : BJOG: An International Journal of Obstetrics \& Gynaecology 2014;121:49-56

11. Temu THB, Masenga G, Obure J, Mosha D, Mahande MJ. Maternal and obstetric risk factors associated with preterm delivery at a referral hospital in northern-eastern Tanzania. Asian Pacific Journal of Reproduction 2016; 5: 365-37

12. Ding X, Wu Y, Xu SH, Zhu R, Jia XM, Zhang $\mathrm{SH}$, et al. Maternal anxiety during pregnancy and adverse birth outcomes: a systematic review and metaanalysis of prospective cohort studies. Journal of Affective Disorders 2014; 20:103-110 
13. Goldenberg RL, Culhane JF, Iams JD, Romero R. Epidemiology and Causes of preterm Birth. The Lancet 2008; 371:75-84

14. Spong CY, Mercer BM, D'Alton M, Kilpatrick S, et al. Timing of indicated late-preterm and early-term birth. Obstetrics and Gynecology 2011;118:323

15. Ghosh J, Wilhelm, M. H, Dunkel-Schetter C, Lombardi C. et al. Paternal support and preterm birth, and the moderation of effects of chronic stress: a study in Los Angeles County mothers. Archives of Women's Mental Health 2010; 13:327-338

16. Khader Y, Al-Shishani L, Obeidat B, Khassawneh M, et al. Maternal periodontal status and preterm low birth weightdelivery: a caseecontrol study. Archives of Gynecology and Obstetrics 2009;279:165-9

17. Trivedi S, Joachim M, McElrath T, Kliman HJ, Allred EN, Fichorova RN, et al. Onderdonk A, Heitor F, Chaychi L, Leviton A, Majzoub JA, Extremely Low Gestational Age Newborns (ELGAN) study investigators. Fetal-placental inflammation, but not adrenal activation, is associated with extreme preterm delivery. American Journal of Obstetrics and Gynecology 2012;206: 327-338

18. Holbrook RH, Laros RK, Creasy RK. Evaluation of a risk-scoring system for prediction of preterm labor. American Journal of Perinatology 1989; $6: 62-8$

19. Rajaeefard A, Mohammadi M, Choobineh A. Preterm delivery risk factors: a prevention strategy in Shiraz, Islamic Republic of Iran. Eastern Mediterranean Health Journal 2007; 13:551-559

20. Rezaeian M, Abedian Z, Latifnejad Roudsari R, Mazloom R, Dadgar S. The relationship of prenatal self-care behaviors with stress, anxiety and depression in women at risk of preterm delivery. The Iranian Journal of Obstetrics, Gynecology and Infertility 2017; 20: $68-76$
21. Ajami ME, Nik Khah Shahmirzadi A, Nik Khah A. Prevalence and factors associated with preterm birth in Shahrood in 2012. Nasim Tandorosti Journal 2012; 2:43-48

22. Roozbeh N, Moradi S, Soltani S, Zolfizadeh F, Hasani MT, Yabandeh AP. Factors associated with preterm labor in Hormozgan province in 2013. Electronic physician 2016; 8: 2918-2923

23. Derakhshi B, Esmailnasab N, Ghaderi E, Hemmatpour S. Risk factor of preterm labor in the west of Iran: a case-control study. Iranian Journal of Public Health 2014; 43: 499-506

24. Wang Y, Liou J, Pan W. Association between maternal periodontal disease and preterm delivery and low birth weight. Taiwanese Journal of Obstetrics and Gynecology 2013; 52:71-76

25. Vettore MV, Leao AT, do Carmo Leal M, Feres M, Sheiham A. The relationship between periodontal disease and preterm low birth weight: clinical and microbiological results. Journal of Periodontal Research 2008; 43:615

26. Pitiphat W, Joshipura KJ, Gillman MW, Williams PL, Douglass CW, Rich-Edwards JW. Maternal periodontitis and adverse pregnancy outcomes. Community Dentistry Oral Epidemiology 2008; 36:3-11

27. Shoja M, Shoja E, Gharaei M. Prevalence and affecting factors on preterm birth in pregnant women Referred to Bentolhoda hospital-Bojnurd. Journal of North Khorasan University of Medical Sciences 2016; 7: 855-863

28. Huang A, Jin X, Liu X, Gao S. A matched case control study of preterm birth in one hospital in Beijing, China. Reproductive Health 2015;12:1 